\title{
Trajectory tracking control of quantum systems
}

\author{
CONG Shuang* \& LIU JianXiu \\ Deptartment of Automation, University of Science and Technology of China, Hefei 230027, China
}

Received November 17, 2011; accepted February 1, 2012; published online May 4, 2012

\begin{abstract}
For quantum state trajectory tracking of density matrix in Liouville equation of quantum systems, with the help of concept in quantum system control, one can apply unitary transformation both to controlled system and free-evolutionary target system such as to change the time-variant and non-stationary target system into a stationary state. Therefore, the quantum state trajectory tracking problem becomes a steering one. State steering control law of the system transformed is designed by means of the Lyapunov stability theorem. Finally, numerical simulation experiments are given for a five-level energy quantum system. The comparison analysis of original system's trajectory tracking with other method illustrates the advantage in control time of the method proposed.
\end{abstract}

trajectory tracking, unitary transformation, free-evolutionary target system, regulation

Citation: Cong S, Liu J X. Trajectory tracking control of quantum systems. Chin Sci Bull, 2012, 57: 2252-2258, doi: 10.1007/s11434-012-5194-z

In recent years, state preparation and steering problem have been extensively studied [1-3] due to their great important applications in quantum teleportation [4], quantum computation and quantum information [5]. Quantum trajectory tracking is of equal importance as quantum state steering. It requires controlled state to be able to follow a time-variant target state, which is more complicated than the control of state steering. On the other hand, the convergence of quantum tracking is as much important as its control law design. The probability of state in a quantum system makes a stable control not enough any more. The asymptotic convergence of control strategy in such a case is needed to make sure that the experiments are practicable. Therefore the convergence of the control of quantum system directly determines whether a proper control law is designed. At the same time the condition of convergence can provide the methods and ways to derive an effective control law. Especially in quantum systems, the complexity of controlled system model, various characteristics of different quantum states and the probability of controlled variables lead to great difficulties in state transferring. Furthermore, non-autonomic system

*Corresponding author (email: scong@ustc.edu.cn) appears in tracking control, all of which show many difficulties in a quantum system's trajectory tracking control.

In [6], with the help of some concepts in system control, we studied trajectory tracking of quantum state: introducing an error state $e(t)$ by defining subtraction between target state $\hat{\rho}_{f}(t)$ and system state $\hat{\rho}(t)$. We changed the tracking control goal of $\hat{\rho}(t)$ following $\hat{\rho}_{f}(t)$ into a regulation problem of error state $e(t)$ from the arbitrary initial error state $e(0)$ to its stable state $e\left(t_{f}\right)$ which is a zero matrix and denoted by $e\left(t_{f}\right)=O$. To design a proper control law, we also introduced a unitary transformation: $U(t)=\exp \left(-i t H_{0}\right)$ to remove the drift item with $H_{0}$ which makes it difficult to determine the sign of the first order derivative of Lyapunov function.

We find that the unitary transformation introduced in [6] can not only eliminate the drift item with $H_{0}$ but also turn the time-dependent target state $\hat{\rho}_{f}(t)$ of original system into a stationary state $\hat{\rho}_{f 0}$. In other words, with the help of the unitary transformation the system state $\hat{\rho}(t)$ tracking target state $\hat{\rho}_{f}(t)$ simultaneously becomes the steering problem of the system state toward a target initial state $\hat{\rho}_{f 0}$ from 
which we think the procedure of introducing error state $e(t)$ is not necessary in quantum tracking control, which motivates this paper.

In this work, the unitary transformation will be applied to the original system, which changes free-evolutionary target system $\hat{\rho}_{f}(t)$ into a time-independent state $\hat{\rho}_{f 0}$. We achieve the tracking goal by steering system initial state $\hat{\rho}_{0}$ to $\hat{\rho}_{f 0}$. In such a case, the stable final state $\hat{\rho}_{f 0}$ of steering problem is no longer a zero matrix, which is much different from the case in [6]. Therefore the design of control law and the proof of convergence are more difficult than that in the former.

\section{Description of system model}

The controlled system in this paper is based on quantum Liouville equation acting on the Hilbert space:

$$
\begin{array}{cc}
i \hbar \frac{\partial}{\partial t} \hat{\rho}(t)=[H, \hat{\rho}(t)], & \hat{\rho}(0)=\hat{\rho}_{0}, \\
i \hbar \frac{\partial}{\partial t} \hat{\rho}_{f}(t)=\left[H_{0}, \hat{\rho}_{f}(t)\right], & \hat{\rho}_{f}(0)=\hat{\rho}_{f 0},
\end{array}
$$

where $H=H_{0}+\sum_{m=1}^{M} f_{m}(t) H_{m} . \quad H_{0}$ and $H_{m}$ represent system's internal (or free) and external (or control) Hamiltonian, respectively, and all of them will be assumed to be time-independent. $f_{m}(t)$ are time-dependent external control fields. We choose the Planck constant $\hbar=1$ for convenience. Eq. (1) is the controlled system and eq. (2) describes the target system which is time-dependent and free-evolutionary.

For systems (1) and (2), the expected control goal is: for arbitrary initial state of system (2), state $\hat{\rho}(t)$ of (1) will follow target system (2). Since the evolution of a closed quantum systems is unitary, the spectrum of $\hat{\rho}(t)$ is timeinvariant, viz. $\operatorname{Tr}\left[\hat{\rho}^{n}(t)\right]=\operatorname{Tr}\left[\hat{\rho}_{f}^{n}(t)\right]$.

The transformation deduced by a linear unitary operator is called unitary transformation. Here we introduce unitary transformation $U(t)=\exp \left(-i t H_{0}\right)$ in original systems (1) and (2), and the controlled state $\hat{\rho}(t)$ and target state $\hat{\rho}_{f}(t)$ become

$$
\begin{aligned}
& \rho(t)=U^{+}(t) \hat{\rho}(t) U(t), \\
& \rho_{f}(t)=U^{+}(t) \hat{\rho}_{f}(t) U(t),
\end{aligned}
$$

where "+" denotes conjugate, $\hat{\rho}(t)$ and $\hat{\rho}_{f}(t)$ are system state and target state before unitary transformation, respectively. Because of $U(0)=U^{+}(0)=1(t=0)$, one has $\rho(0)=$ $\hat{\rho}(0)=\hat{\rho}_{0}, \rho_{f}(0)=\hat{\rho}_{f}(0)=\hat{\rho}_{f 0}$ after transformation. More- over, target system (2) is free-evolutionary under free Hamiltonian $H_{0}$. The solution of (2) is $\hat{\rho}_{f}(t)=U(t) \hat{\rho}_{f}(0) U^{+}(t)$ where $U(t)$ is the evolution matrix and $U(t)=\exp \left(-i t H_{0}\right)$. Substituting this solution into (3), the transformed target state $\rho_{f}(t)$ becomes

$$
\rho_{f}(t)=U^{+}(t) U(t) \hat{\rho}_{f}(0) U^{+}(t) U(t)=\hat{\rho}_{f}(0)=\hat{\rho}_{f 0} .
$$

Therefore the original systems (1) and (2) after unitary transformation will be

$$
\begin{gathered}
i \hbar \frac{\partial}{\partial t} \rho(t)=\left[\sum_{m} f_{m}(t) H_{m}(t), \rho(t)\right], \quad \rho(0)=\hat{\rho}_{0}, \\
\rho_{f}(t)=\hat{\rho}_{f 0} .
\end{gathered}
$$

Eq. (5) is the new controlled and target systems from which one can see that:

Remark 1. State $\hat{\rho}_{f}(t)$ in original target system (2) evolves according to Liouville equation, which is time-dependent and non-stationary. After unitary transformation the trajectory of target system in (5) becomes time-independent and stationary which is a constant value and equals to the initial state $\hat{\rho}_{f 0}$ of target system.

Remark 2. After the transformation the control Hamiltonian $H_{m}$, which is time-independent, becomes $H_{m}(t)$ with time-dependent and determines the evolution of states in interaction picture.

Unitary transformation in this paper has two meanings, one of which is the rotation of the basic vectors in Hilbert space. We say a unitary operator leads to the rotation of the Hilbert space. New state operators $\rho(t)$ and $\rho_{f}(t)$ are used to describe the transformed quantum system (5). Under unitary transformation, all physical properties are unchanged. The other meaning is that only control Hamiltonian remained in new system (5a) makes every item in the first order derivation of Lyapunov function controllable, which is convenient to design control law.

By introducing unitary transformation, the original trajectory tracking problem of system state $\hat{\rho}(t)$ following freeevolutionary target state $\hat{\rho}_{f}(t)$ becomes the steering problem of state $\rho(t)$ being regulated to $\hat{\rho}_{f}(0)$ in system (5).

\section{Design of control law}

Among many control methods, the Lyapunov-based method is simple and easy to design. The basic idea of Lyapunovbased method is to select $V(x)$ as Lyapunov function, which must satisfy the following three conditions: (i) $V(x)$ is continuous and its first-order partial derivatives is also continuous in its definition; (ii) $V(x)$ is positive semi-definite, i.e. $V(x) \geqslant 0$; (iii) The first order time derivative of the Lyapunov 
function is negative semi-definite, i.e. $\dot{V}(x) \leqslant 0$. If they are true then the control system is stable. In system control field one often uses the Lyapunov-based method to design the control law because this controller design method can guarantee the whole control system is stable. We will use the Lyapunov-based method next.

Select the average value of an observable quantity as the Lyapunov function here

$$
V=\operatorname{tr}(P \rho)+C
$$

where $P$ is the observable operator, and it is not necessarily positive definite as it not always corresponds to an actual observable quantity. $P$ is also called a virtual mechanical quantity operator. $C$ is a constant used to adjust the value of Lyapunov function.

According to the third condition of Lyapunov function and system (5), one can obtain

$$
\dot{V}=-\sum_{m} f_{m}(t) \operatorname{tr}\left(i H_{m}(t)[\rho(t), P]\right) .
$$

For the sake of simplicity and availability we let each item in the right side of (7) of summation sign be non-positive in order to ensure $\dot{V} \leqslant 0$. The control law can be derived as

$$
f_{m}(t)=k_{m} t r\left(i H_{m}(t)[\rho(t), P]\right), \quad k_{m}>0,
$$

where $k_{m}>0$ is the control gain which is used to adjust the convergence speed of the system state.

Generally, control law (8) is only a stable control and cannot always guarantee the system converges to its target state. Because of the probability control in quantum system, only asymptotic stable control can guarantee the system to achieve the target state completely. Next we will study the conditions of convergence of the control law (8).

Firstly, an appropriate observable $P$ will be constructed to ensure the stable target state $\hat{\rho}_{f}(0)$ to be the minimum point of (6).

One can rewrite the condition (ii) and (iii) as

$$
\begin{gathered}
V\left(\rho_{f}\right)=\min _{\rho} V(\rho), \\
\dot{V}\left(\rho_{f}\right)=0 .
\end{gathered}
$$

These conditions ensure a specific form of $P$. In those cases the target state is a stable point in sense of Lyapunov. According to $(9 \mathrm{~b})$, states $\rho(t)$, which induce the control fields $f_{m}(t)$ to be zeros, belong to the set

$$
\mathbb{R} \equiv\left\{\rho: \operatorname{tr}\left(i H_{m}(t)[\rho, P]\right)=0, \forall m, t\right\} .
$$

Different from the method of introducing error state in [6], where stable error state is always zero matrix, the method in this paper is easy to get the condition of operator
$P$. However no clear information can be obtained from (9). The coherent vectors will be used here to construct observable operator $P$.

Rewrite (9) as

$$
\begin{gathered}
\operatorname{tr}\left(P \rho_{f}\right)=\min _{\rho} V(\rho), \\
\rho_{f} \in \mathbb{R} .
\end{gathered}
$$

A Hermite matrix is decomposed by means of a unitary Lie algebra. In such a way, a matrix is expressed as coherent vectors. The bases of a unitary Lie algebra are described as

$$
\left\{X_{1}, \cdots X_{m}\right\}
$$

And the following conditions hold

$$
\begin{gathered}
\operatorname{tr}\left(X_{l}\right)=0, \\
\operatorname{tr}\left(X_{l} X_{j}\right)=\delta_{l j} .
\end{gathered}
$$

For $\forall l, \exists \gamma_{j}, \gamma_{k} \neq 0$, one has

$$
\left[\sum_{j} \gamma_{j} X_{j}, \sum_{k} \gamma_{k} X_{k}\right]=i X_{l} .
$$

Then an $n$-dimensional state density matrix can be rewritten as

$$
\rho=I / n+\sum x_{l} X_{l}
$$

In such a way, the coherent vector $R_{\rho}$ of a density matrix $\rho$ can be expressed as

$$
R_{\rho}=\left(x_{1}, x_{2}, \cdots x_{n^{2}-1}\right)^{T} .
$$

The module of coherent vector indicates the purity of density matrix, which reaches its maximum $\sqrt{(n-1) / n}$ when $\rho$ is pure state and zero of maximum mixed-state $I / n$.

Because of the unitary characteristic of closed quantum system, the purity in evolution is unchanged, then at any time it is satisfied

$$
\sum_{l}\left|x_{l}\right|^{2}=C, C \text { is a constant. }
$$

We assume that system state and target state both are controllable in this paper.

According to the theoretical analysis mentioned above, the target state and observable operator $P$ are unfolded as follows:

$$
\begin{gathered}
\rho_{f}=I / n+\sum_{j} f_{j} X_{j}, \\
P=c_{0} I+\sum_{k} c_{k} X_{k} .
\end{gathered}
$$


Substituting (17) into (11), one has

$$
\operatorname{tr}\left(P \rho_{f}\right)=c_{0}+\sum_{k} c_{k} f_{k}
$$

If we want to get the minimum point of (18), then

$$
c_{k}=\lambda f_{k}, \lambda<0
$$

which implies coherent vector $R_{P}$ of operator $P$ is opposite in direction from that of target state $\rho_{f}$ :

$$
R_{P}=\lambda R_{\rho_{f}}, \lambda<0 .
$$

Therefore the simplest method to construct the observe operator $P$ is to choose

$$
P=-\rho_{f} .
$$

The structure of $P$ in (21) is same as that in [6] which ensures $\rho_{f}$ is the minimum point of Lyapunov function (6) and control law (8) is stable.

The other procedure of convergence proof is the same of that in [7]. We do not repeat it here.

\section{Numerical simulation experiments and results analysis}

In order to demonstrate the effectiveness and advantage of the method proposed in this paper, two examples and results analysis will be given in this section.

A five-level energy quantum system used both in [8] and [9] is selected, where the free Hamiltonian is

$$
H_{0}=\left(\begin{array}{ccccc}
1 & 0 & 0 & 0 & 0 \\
0 & 1.2 & 0 & 0 & 0 \\
0 & 0 & 1.3 & 0 & 0 \\
0 & 0 & 0 & 2 & 0 \\
0 & 0 & 0 & 0 & 2.15
\end{array}\right)
$$

Eigenvalues of $H_{0}$ are $\lambda_{1}=1, \lambda_{2}=1.2, \lambda_{3}=1.3, \lambda_{4}=2, \lambda_{5}=2.15$. The corresponding eigenstates are

$$
\left|\lambda_{1}\right\rangle=\left[\begin{array}{l}
1 \\
0 \\
0 \\
0 \\
0
\end{array}\right],\left|\lambda_{2}\right\rangle=\left[\begin{array}{l}
0 \\
1 \\
0 \\
0 \\
0
\end{array}\right],\left|\lambda_{3}\right\rangle=\left[\begin{array}{l}
0 \\
0 \\
1 \\
0 \\
0
\end{array}\right],\left|\lambda_{4}\right\rangle=\left[\begin{array}{l}
0 \\
0 \\
0 \\
0 \\
1
\end{array}\right] \text { and }\left|\lambda_{5}\right\rangle=\left[\begin{array}{l}
0 \\
0 \\
0 \\
0 \\
1
\end{array}\right] \text {. }
$$

It is assumed that initial state of system (1) is $\hat{\rho}_{0}$. The freeevolutionary target system (2) has an initial value $\hat{\rho}_{f 0}$.

According to the conditions mentioned above, after unitary transformation new system (5) replaces the original systems (1) and (2). The tracking problem has become state steering one with new state $\rho(t)$ in system (5) from its ini- tial state $\hat{\rho}_{0}$ to a stationary target state $\hat{\rho}_{f 0}$.

In this system, all the transition frequencies are different viz. $\omega_{j k} \neq \omega_{p q},(j, k) \neq(p, q)$ where $\omega_{j k}=\lambda_{j}-\lambda_{k}$, which satisfies assumption 1 of convergence conditions in [7]. According to assumption 2, control Hamiltonians must be full connected, which means ten control Hamiltonians are needed for this system. However in most cases, we do not need so many control Hamiltonians, the number of which is determined by the concrete structure of $\hat{\rho}_{0}$ and $\hat{\rho}_{f 0}$.

Example A. The initial state of system (1) is an eigenstate

$$
\left|\lambda_{1}\right\rangle=\left[\begin{array}{c}
1 \\
0 \\
0 \\
0 \\
0
\end{array}\right] \text { of } H_{0} \text {, then } \hat{\rho}_{0}=\left(\begin{array}{ccccc}
1 & 0 & 0 & 0 & 0 \\
0 & 0 & 0 & 0 & 0 \\
0 & 0 & 0 & 0 & 0 \\
0 & 0 & 0 & 0 & 0 \\
0 & 0 & 0 & 0 & 0
\end{array}\right) \text {. }
$$

The probability superposition of eigenstate $\left|\lambda_{2}\right\rangle$ and $\left|\lambda_{3}\right\rangle$ is the initial state of free-evolutionary target system (2), viz. $\left|\psi_{f 0}\right\rangle=1 / \sqrt{2}\left|\lambda_{2}\right\rangle+1 / \sqrt{2}\left|\lambda_{3}\right\rangle$

$$
\hat{\rho}_{f 0}=\left|\psi_{f 0}\right\rangle\left\langle\psi_{f 0}\right|=0.5 \times\left(\begin{array}{ccccc}
0 & 0 & 0 & 0 & 0 \\
0 & 1 & 1 & 0 & 0 \\
0 & 1 & 1 & 0 & 0 \\
0 & 0 & 0 & 0 & 0 \\
0 & 0 & 0 & 0 & 0
\end{array}\right) .
$$

Considering the specific forms of $\hat{\rho}_{0}$ and $\hat{\rho}_{f 0}$, the density matrixes of initial state and target state only have differences in the population of energies 1,2 and 3 and the interference item of energies 2 and 3. So the designed control law is aimed to these different items, that is to say, interaction only existed between energies 1, 2 and 3. Three control Hamiltonians are enough to deal with the problem in this section shown as

$$
\begin{aligned}
H_{1} & =\left(\begin{array}{lllll}
0 & 1 & 0 & 0 & 0 \\
1 & 0 & 0 & 0 & 0 \\
0 & 0 & 0 & 0 & 0 \\
0 & 0 & 0 & 0 & 0 \\
0 & 0 & 0 & 0 & 0
\end{array}\right), H_{2}=\left(\begin{array}{lllll}
0 & 0 & 1 & 0 & 0 \\
0 & 0 & 0 & 0 & 0 \\
1 & 0 & 0 & 0 & 0 \\
0 & 0 & 0 & 0 & 0 \\
0 & 0 & 0 & 0 & 0
\end{array}\right), \\
H_{3} & =\left(\begin{array}{lllll}
0 & 0 & 0 & 0 & 0 \\
0 & 0 & 1 & 0 & 0 \\
0 & 1 & 0 & 0 & 0 \\
0 & 0 & 0 & 0 & 0 \\
0 & 0 & 0 & 0 & 0
\end{array}\right) .
\end{aligned}
$$

Next, we consider the diagonal elements $\rho_{n n}(n=1,2 \ldots .5)$ of density matrix which is the population of state, and offdiagonal elements $\rho_{23}$, which represents interference item. 
Here we mainly observe the tracking of interference item $\rho_{23}$.

According to (22), the control fields corresponding to each Hamiltonian are $f_{1}, f_{2}$ and $f_{3}$, respectively. Control law can be obtained from (8) where $k=0.5, f_{0}=0.015$.

To demonstrate the tracking of system (1) to system (2) we keep data of control function $f_{m}(t), m=1,2,3$ at all times and apply them to original system (1). It is known that the population of target system (2) is unchanged under unitary evolution. Figure 1 displays the effectiveness of state steering of $\rho(t)$ from its initial state $\hat{\rho}_{0}$ to final state $\hat{\rho}_{f 0}$ of system (5), in which Figure 1(a) is the evolution of diagonal items $\rho_{n n}, n=1,2,3$ and interference item $\rho_{23}$ of state $\rho(t)$.

From Figure 1(a) one can see that both of them evolves towards relevant items of target state $\hat{\rho}_{f 0}$. Figure 1(b) is the tracking of interference item in which dot line is freeevolutionary trajectory of $\hat{\rho}_{f 23}$ of target system state $\hat{\rho}_{f}(t)$ and solid line is tracking curve of $\hat{\rho}_{23}$ of controlled system state $\hat{\rho}(t)$. Figure $1(\mathrm{c})$ shows the control fields $f_{m}(t)$. We set a performance index to describe the distance between initial state and target state: $v=\left\|\rho_{f}(t)-\rho(t)\right\|^{2}$. Here, $v=1.4125 \times$ $10^{-6}(t=30$ a.u. $)$.

Example B. We choose the same example as in [8], where the initial state of system (1) is a superposition state $\left|\psi_{0}\right\rangle=1 / \sqrt{3}\left|\lambda_{1}\right\rangle+\sqrt{2} / \sqrt{3}\left|\lambda_{4}\right\rangle$. The initial target state of system (2) is an eigenstate $\left|\lambda_{3}\right\rangle=[0,0,1,0,0]^{T}$.

Here we choose the same parameters $k=0.15$ and

$$
P=\left(\begin{array}{ccccc}
0.8 & 0 & 0 & 0 & 0 \\
0 & 1.1 & 0 & 0 & 0 \\
0 & 0 & 0.4 & 0 & 0 \\
0 & 0 & 0 & 1.2 & 0 \\
0 & 0 & 0 & 0 & 0.6
\end{array}\right)
$$

as those in [8], but a different control Hamilton is selected of the form:

$$
H_{c}=\left(\begin{array}{lllll}
0 & 0 & 1 & 0 & 0 \\
0 & 0 & 0 & 0 & 0 \\
1 & 0 & 0 & 1 & 0 \\
0 & 0 & 1 & 0 & 0 \\
0 & 0 & 0 & 0 & 0
\end{array}\right),
$$

where interactions exist between energies 1 and 3 and between energies 4 and 3, respectively. The simulation experimental results are shown in Figure 2, which demonstrates the effectiveness of state steering of $\rho(t)$ from its initial state $\hat{\rho}_{0}$ to the final one $\hat{\rho}_{f 0}$. Figure 2(a) shows the population evolution, Figure 2(b) is the control law and Figure 2(c) shows the interference item $\rho_{14}$ in which dashed
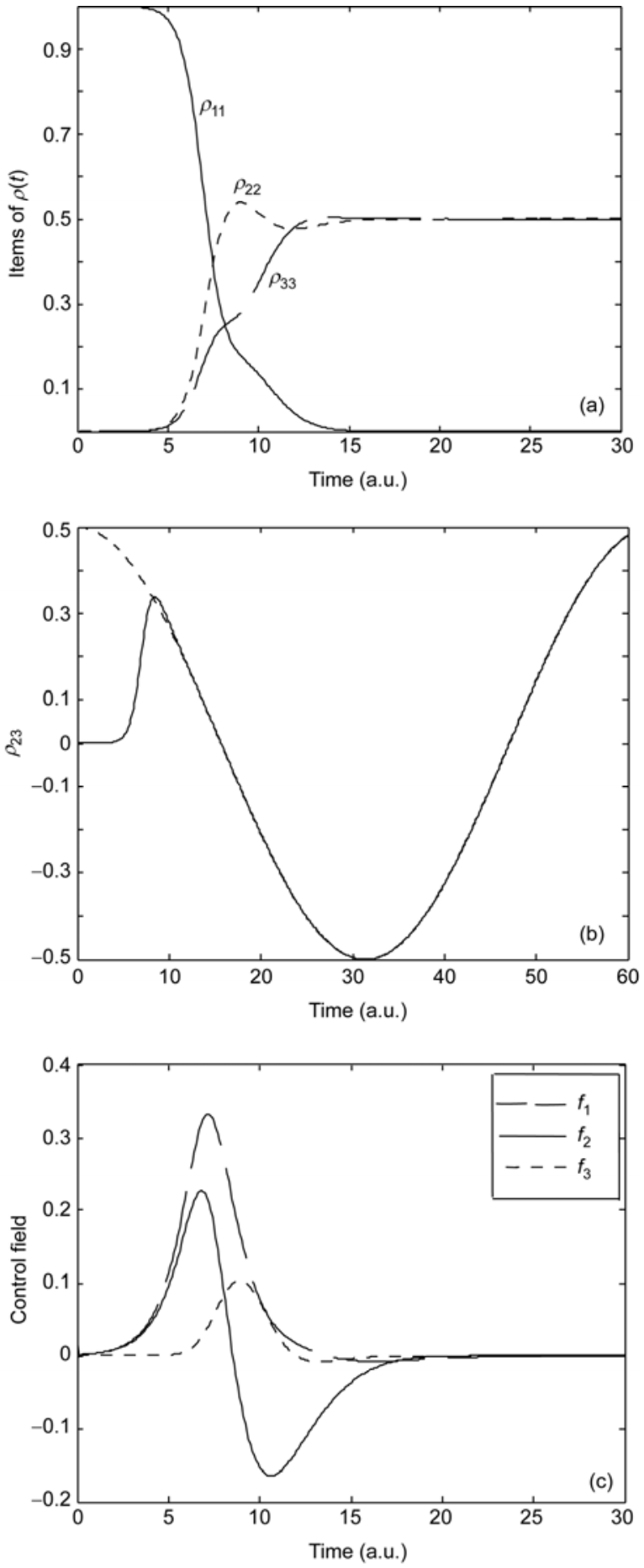

Figure 1 (a) Population of state $\rho(t)$ in system (5); (b) tracking of $\rho_{23}$ for original systems (1) and (2); (c) control fields $f_{m}(t)$.

line indicates the item of target state and solid line indicates the corresponding item of $\rho(t)$.

To achieve the same goal one needs a control time of 400 a.u. in [8], but form Figure 2 one can see that here a different control Hamiltonian designed results in a shorter time of less than 150 a.u.

In experiment $\mathrm{B}$, we can divide the control Hamiltonian 

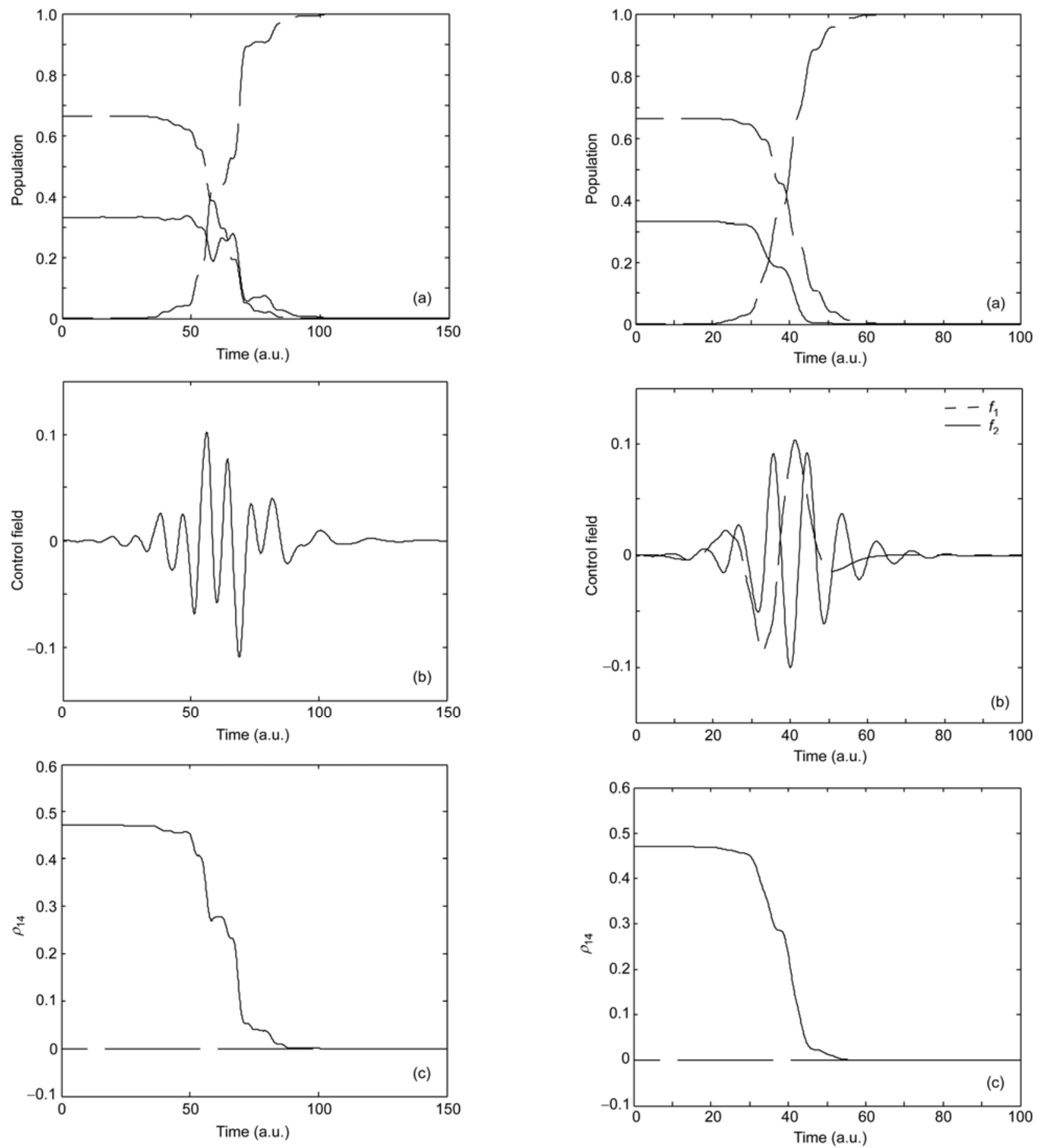

Figure 2 (a) Population of state $\rho(t)$ in system (5); (b) control fields $f_{1}(t)$; (c) tracking of interference item $\rho_{14}$.

Figure 3 (a) Population of state $\rho(t)$ in system (5); (b) control fields $f(t)$; (c) tracking of interference item $\rho_{14}$.

into two parts, as in experiment A. If we set

$$
H_{1}=\left(\begin{array}{lllll}
0 & 0 & 1 & 0 & 0 \\
0 & 0 & 0 & 0 & 0 \\
1 & 0 & 0 & 0 & 0 \\
0 & 0 & 0 & 0 & 0 \\
0 & 0 & 0 & 0 & 0
\end{array}\right), H_{2}=\left(\begin{array}{lllll}
0 & 0 & 0 & 0 & 0 \\
0 & 0 & 0 & 0 & 0 \\
0 & 0 & 0 & 1 & 0 \\
0 & 0 & 1 & 0 & 0 \\
0 & 0 & 0 & 0 & 0
\end{array}\right),
$$

where $H_{c}=H_{1}+H_{2}$. Then two control fields will be needed. The results are shown in Figure 3, where the three figures correspond to the ones in Figure 2. We can see the time to complete control task is shorter than that in Figure 2, which is less than 100 a.u. In Figure 2, there is only one control Hamiltonian included all energy transforms, so control action on all energy is the same, which brings restrictions to control fields and lengthens the time to complete control 
task, sometimes even causes larger error.

\section{Conclusion}

In this paper we have developed unitary transformation to change tracking problem of quantum state into steering one, which leads to the same results as in [6]. The special unitary transformation makes tracking problem in closed quantum system easier than that in classical one. If a closed quantum system is considered, the tracking problem of a free-evolutionary quantum system, which is a very common situation, becomes the regulation of controlled system initial state to target system initial state. It is a special case of the tracking problem. The error state is not necessary. But it is not the case for a non-free-evolutionary target system, such as a controlled target system and a predefined target time-dependent function, or in open quantum system $[10,11]$, which need to use the method in [6]. We also demonstrate that different structure of control Hamiltonians, which in fact describes different control paths such as the comparison analysis between experiment B-2 and [8], affects control time. The control Hamiltonians with same structure, such as experiment B-2 and B-3 in this paper, may have shorter control time when they are organized separately instead of as a whole.
This work was supported in part by the National Natural Science Foundation of China (61074050) and the Doctoral Fund of Ministry of Education of China (20103402110044).

1 Cong S, Zhang Y Y. Superposition state preparation based on Lyapunov stability theorem in quantum systems. J Univ Sci Tech China, 2008, 38: 821-827

2 Resch K, Lundeen J, Steinberg A. Quantum state preparation and conditional coherence. Phys Rev Lett, 2002, 88: 113601

3 Shi T, Li Y, Song Z, et al. Quantum-state transfer via the ferromagnetic chain in a spatially modulated field. Phys Rev A, 2005, 71: 032309

4 Wang X. Quantum teleportation of entangled coherent states. Phys Rev A, 2001, 64: 022302

5 Sillanpää M A, Park J I, Simmonds R W. Coherent quantum state storage and transfer between two phase qubits via a resonant cavity. Nature, 2007, 449: 438-442

6 Liu J X, Cong S. Trajectory tracking of quantum states based on Lyapunov method. In: The 9th IEEE International Conference on Control \& Automation, Santiago, Chile, 2011. 318-323

7 Lou Y S, Cong S, Yang J, et al. Path programming control strategy of quantum state transfer. IET Control Theory Appl, 2011, 5: 291-298

8 Kuang S, Cong S, Lou Y S. Population control of quantum states based on invariant subsets under a diagonal Lyapunov function. In: Joint 48th IEEE Conference on Decision and Control and 28th Chinese Conference, Shanghai, 2009. 2486-2491

9 Ramakrishna V, Salapaka M, Dahleh M, et al. Controllability of molecular systems. Phys Rev A, 1995, 51: 960-966

10 Rong X, Wang Y, Yang J H, et al. Dynamical decoupling of electron spins in phosphorus-doped silicon. Chin Sci Bull, 2011, 56: 591-597

11 Cao W C, Liu X S, Bai H B. Bang-bang control suppression of amplitude damping in a three-level atom. Sci China Ser G-Phys Mech Astron, 2008, 51: 29-37

Open Access This article is distributed under the terms of the Creative Commons Attribution License which permits any use, distribution, and reproduction in any medium, provided the original author(s) and source are credited. 